\title{
Electromagnetic Characterization of Aircraft Composite Materials and its Effects on the Antenna Performance
}

L. G. da Silva' ${ }^{1}$, I. A. Baratta ${ }^{2}$, R. R. de Assis ${ }^{2}$, L. N. Bellei ${ }^{2}$, C. B. de Andrade' 2 P. P. F. Campici ${ }^{2}$, S. O. Nunes ${ }^{2}$, M. C. Paiva ${ }^{1}$ and Arismar Cerqueira S. Jr. ${ }^{1}$

\author{
${ }^{1}$ Inatel Competence Center \\ National Institute of Telecommunications \\ Santa Rita do Sapucaí, Brazil \\ luis.gustavo@inatel.br andarismar@inatel.br \\ ${ }^{2} R \& D$ \\ Embraer S.A \\ São José dos Campos, Brazil \\ sidney@embraer.com.br
}

\begin{abstract}
This paper presents an electromagnetic characterization of aircraft composite materials, as well as numerical and experimental analyses of its effects on the antenna performance. Two uncalibrated S-parameters characterization methods have been applied for retrieving the complex electrical permittivity in the L- and C-band, namely: Air-region method and Sample-shifted method. Dielectric constants of 4.6 and 1.84 and loss tangent of $2.0 \times 10^{-2}$ and $6.1 \times 10^{-2}$ have been obtained for fiberglass and honeycomb composite materials, respectively. A 3.0-meters prototype of an Embraer light jet aircraft dorsal fin was fabricated and used in the experiments in a semi-anechoic chamber in order to evaluate the impact of installing aeronautical VHF and L-band antennas on a fuselage made of composite materials.
\end{abstract}

Index Terms - Aircraft, antenna, composite material and material characterization.

\section{INTRODUCTION}

Electromagnetic characterization of the aircraft structures is an important part of the computeraided engineering simulations, since composite materials are extensively used in aeronautical applications. For this reason, an accurate definition of the materials electromagnetic properties [1], especially non-metallic composite materials, becomes essential to achieve high reliability in the numerical analysis, which contributes considerably to the aircraft development. Furthermore, the possibility of installing antennas under these materials is a reality, since the number of wireless systems and radars has been increased in the last years. Fig. 1 presents an antenna installation under an aircraft composite structure. The composite material could impact on the antenna performance and, consequently, change its electromagnetic properties such as impedance matching, operational bandwidth, radiation pattern, gain and so on. Therefore, numerical analyses of the material properties and antenna performance are highly recommended in order to proper define the antenna placement 
according to the aeronautical constraints.

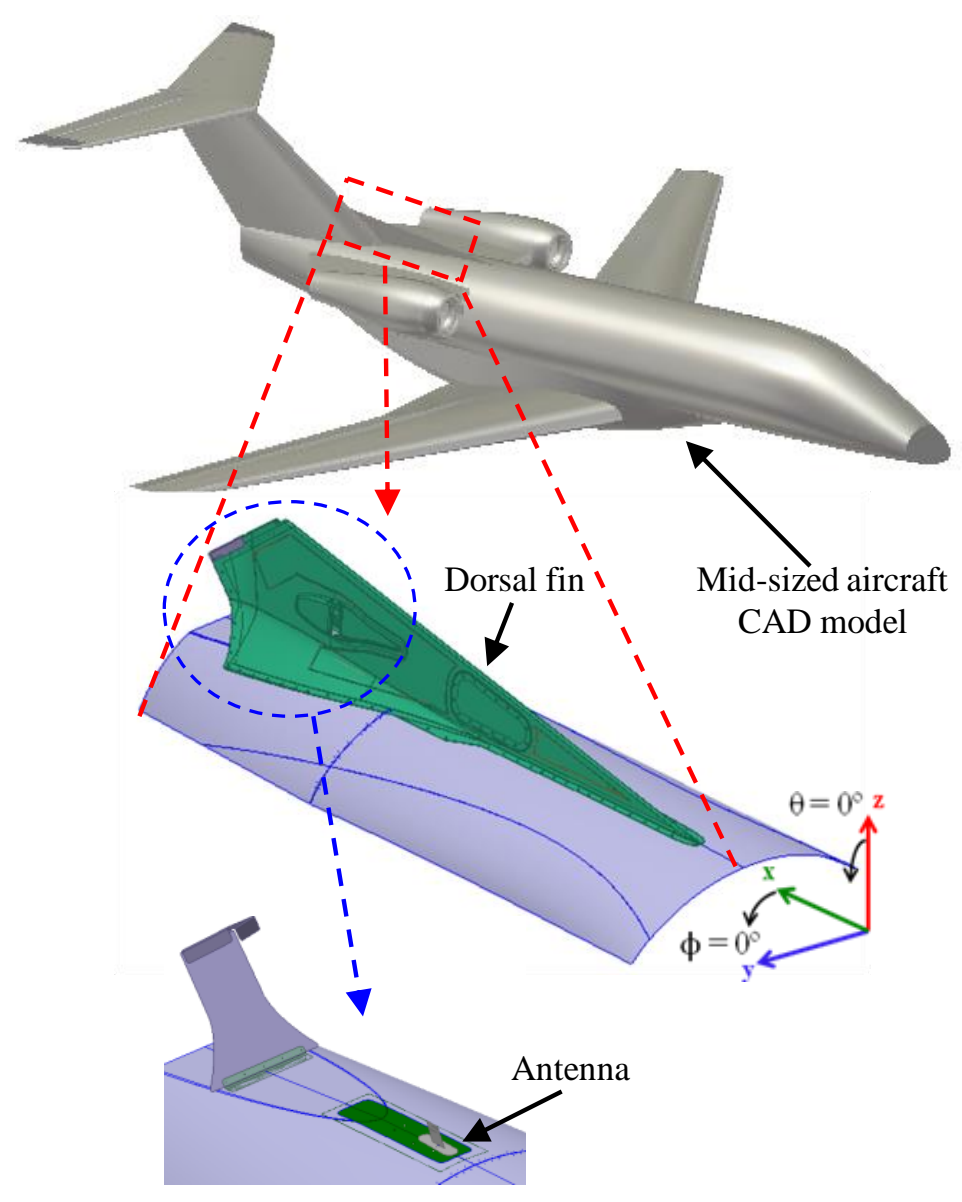

Fig. 1. A mid-sized aircraft and the HFSS numerical model of its dorsal fin.

This work presents an electromagnetic characterization of fiberglass and honeycomb composite materials used in airframe structures. The manuscript is structured in five sections. Section II describes the material characterization based on two uncalibrated S-parameters iterative methods, namely: Air-region method and Sample-shifted method. Section III presents the material characterization results, whereas the performance analysis of two commercial antennas embedded onto a real-size dorsal fin is reported in Section IV. Conclusions and future works are highlighted in Section V.

\section{COMPosite Material Electromagnetic Characterization}

Material characterization methods are categorized into resonant and non-resonant methods. The non-resonant methods are preferred for applications with broadband frequency characterization, besides requiring less sample preparation than resonant methods [2]. In contrast, resonant methods have better accuracy and sensitivity when compared to non-resonant ones, but it suffers from narrow bandwidth results and sample preparation procedures [3]. Additionally, characterization methods can be categorized into calibration-dependent and calibration-independent methods. The well-known calibration-dependent method Nicolson-Ross-Wier (NRW) is a non-iterative approach that compute 
material complex permeability and permittivity using measured S-parameters in a proper setup test [4][5]. The NRW method presents either instability, if the scattering parameters $S_{11}$ and $S_{21}$ approaching zero, or phase uncertainties, when samples thickness are integer multiples of one-half wavelength [6]. Different approaches have been proposed to overcome NRW drawbacks. Boughriet et al. introduced effective permittivity and permeability parameters concept [7]; Barker-Javis et al. proposed an iterative procedure with non-magnetic material assumption [8]; Chalapat et al. combined NRW method and Barker-Jarvis technique into an explicit and reference-plane invariant methodology [9]; and so forth.

There is a growing trend in calibration-independent methods due to its advantages of eliminating calibration standards imperfections and reducing the overall measurement time [10]. These methods typically evaluate the material complex permittivity based on different measurement steps and iterative approach, such as: different sample lengths with and without error-correction, as described in [11] and [12], respectively. In [13] is proposed a two-measurement step technique with a shifted and unshifted sample inside a measurement cell. Sample-shifted method presented in [10] can overcome imprecise sample position problem present in [13]. An extra cell insertion approach between measurement cell extremities is reported in [2], and an improved method with extra cells lengths manipulation to avoid singularities issues is shown in [3].

We have applied two different methods for determining the composite materials complex permittivity. First, a method base on air region insertions between the material under test (MUT) and a second with sample-shifted measurements. These methods concepts and calculation procedure are described below.

\section{A. Air-region method}

In Fig. 2 is presented the air-region method setup, which is composed by the following pieces: two waveguide to coax transitions (X and Y); a measurement cell $\left(L_{g}\right)$; two extra cells ( $L_{03}$ and $\left.L_{04}\right)$; and the MUT $(L)$. The measurement cell has transversal dimensions identical to waveguide to coax transition and length $L_{g}$. This method is based on two step uncalibrated S-parameters measurement for obtaining the MUT complex permittivity in an iterative way. In step (a), the MUT is positioned inside the measurement cell between two air regions with lengths $L_{01}$ and $L_{02}$, respectively, and, then, the full two-port S-parameters are extracted. Lastly, two extra cell are inserted in step (b) with $L_{03}$ and $L_{04}$ air regions lengths. These extra cells are inserted as depicted in Fig. 2. After extracting full two-port S-parameters in step (b), MUT complex permittivity can be determined using iterative analysis.

It is assumed that only the dominant mode $\left(\mathrm{TE}_{10}\right)$ is guided through the waveguide measurement cell. The measurement setup is mathematically modeled using the wave cascading matrix (WCM) method [10-12]. The two ports of WCM matrices are defined as $T_{X}, T_{Y}, T_{01}$ to $T_{04}$ and $T_{L}$ for transitions $\mathrm{X}$ and $\mathrm{Y}$, air region waveguide section and sample-filled waveguide section, respectively. Fig. 2 describes the measurement steps by the following two-port WCM matrices: 


$$
M_{a}=T_{X} T_{01} T_{L} T_{02} T_{Y}, \quad M_{b}=T_{X} T_{03} T_{01} T_{L} T_{02} T_{04} T_{Y}
$$

where $M_{a}$ and $M_{b}$ correspond to the measurements steps (a) and (b), respectively.

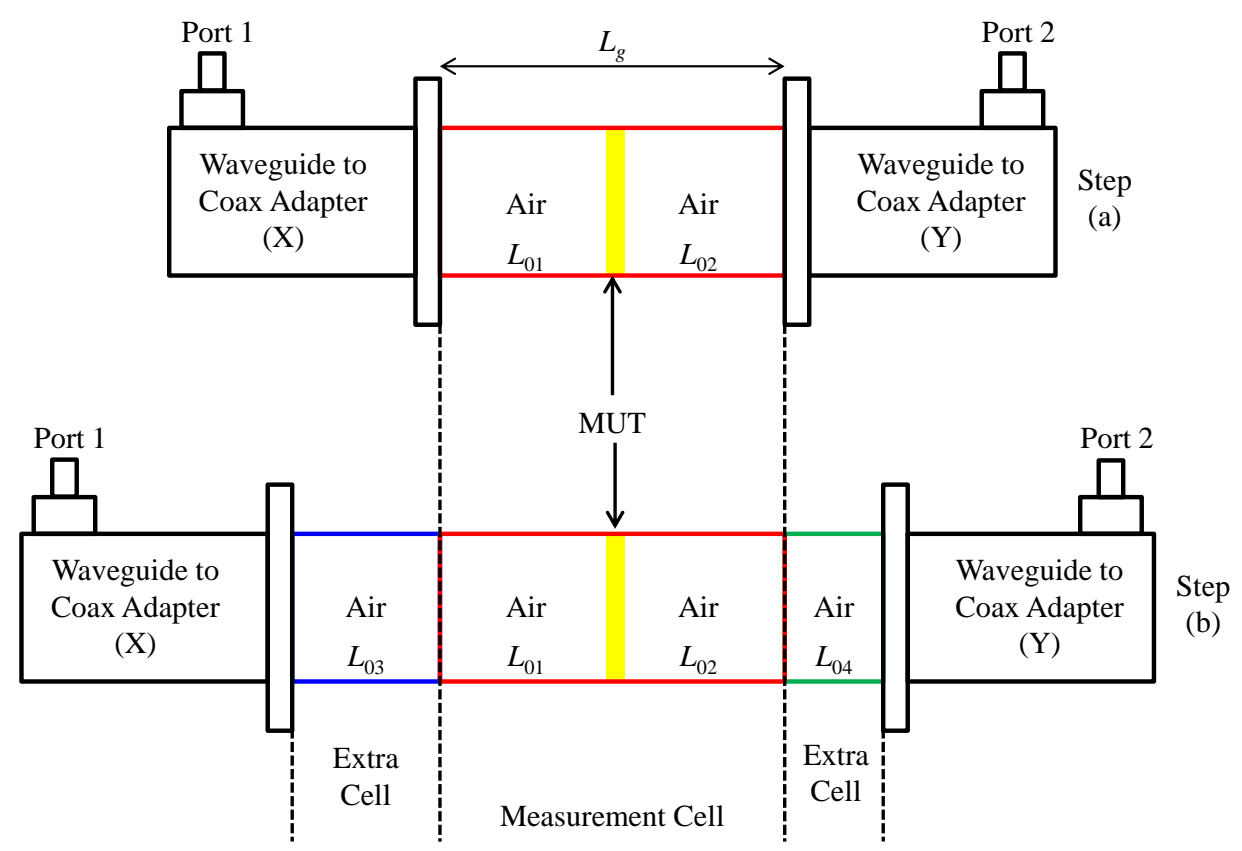

Fig. 2. The air-region method setup.

The WCM matrices and measured uncalibrated S-parameters are related by [12]:

$$
M_{t}=\frac{1}{S_{21 t}}\left[\begin{array}{cc}
\left(S_{12 t} S_{21 t}-S_{11 t} S_{22 t}\right) & S_{11 t} \\
-S_{22 t} & 1
\end{array}\right], t=a, b
$$

where subscripts $a$ and $b$ represent the measurements steps. The air region sections are considered isotropic and nonreflecting line with WCM matrix defined by:

$$
\begin{gathered}
T_{0 n}=\left[\begin{array}{cc}
\alpha_{n} & 0 \\
0 & 1 / \alpha_{n}
\end{array}\right], \alpha_{n}=\mathrm{e}^{-\gamma_{0} L_{0 n}}, n=3,4 \\
\gamma_{0}=i \frac{2 \pi}{\lambda_{0}} \sqrt{1-\left(\frac{\lambda_{0}}{\lambda_{\mathrm{c}}}\right)^{2}}
\end{gathered}
$$

In (3), $\gamma_{0}$ is the air-filled waveguide propagation constant, $\lambda_{0}$ is free-space wavelength and $\lambda_{c}$ is the cut-off wavelength. Now, considering a MUT with isotropic and reciprocal properties, the $T_{L}$ theoretical two-port S-parameters can be written as [10]: 


$$
\begin{gathered}
T_{L}=\frac{1}{\left(1-\Gamma^{2}\right) \mathrm{T}}\left[\begin{array}{cc}
\mathrm{T}^{2}-\Gamma^{2} & \Gamma\left(1-\mathrm{T}^{2}\right) \\
-\Gamma\left(1-\mathrm{T}^{2}\right) & 1-\Gamma^{2} \mathrm{~T}^{2}
\end{array}\right] \\
T_{L}=\left[\begin{array}{cc}
\Lambda_{1} & \Lambda_{2} \\
-\Lambda_{2} & \Lambda_{3}
\end{array}\right]
\end{gathered}
$$

for simplification purpose, we defined the symbols $\Lambda_{1}, \Lambda_{2}$ and $\Lambda_{3}$. The first reflection $(\Gamma)$ and transmission $(\mathrm{T})$ coefficient of sample-filled measurement cell are expressed by:

$$
\Gamma=\frac{\gamma_{0}-\gamma}{\gamma_{0}+\gamma}, \quad \mathrm{T}=\mathrm{e}^{-\gamma L}, \quad \gamma=i \frac{2 \pi}{\lambda_{0}} \sqrt{\varepsilon-\frac{\lambda_{0}}{\lambda_{c}}}
$$

where $\gamma$ is sample-filled waveguide propagation constant and $\varepsilon$ is the MUT complex permittivity. The matrices $T_{X}, T_{Y}, T_{01}$ and $T_{02}$ will be eliminated during the calculation procedure and their expressions can be omitted. A relation between the two measurements steps can be defined using (1), then the following expression can be derived:

$$
M_{b} M_{a}^{-1}=T_{X} T_{03} T_{01} T_{L} T_{02} T_{04} T_{02}^{-1} T_{L}^{-1} T_{01}^{-1} T_{X}^{-1}
$$

in (6) the influence of $T_{Y}$ is eliminated. Analyzing (6), $M_{b} M_{a}^{-1}$ and $T_{03} T_{L} T_{04} T_{L}^{-1}$ are similar matrices and have same trace [14]. Matrix trace is defined in a square matrix as the sum of diagonal elements. Therefore, an expression that relates theoretical and measured S-parameters can be derived by combining (3), (4) and similar matrices trace in (6) to determine MUT complex permittivity.

$$
\left(\frac{\mathrm{T}-1 / \mathrm{T}}{\Gamma-1 / \Gamma}\right)^{2}=\frac{1+\alpha_{3}{ }^{2} \alpha_{4}{ }^{2}-\alpha_{3} \alpha_{4} \operatorname{Tr}\left(M_{b} M_{a}{ }^{-1}\right)}{\alpha_{3}{ }^{2}+\alpha_{4}{ }^{2}-\alpha_{3}{ }^{2} \alpha_{4}{ }^{2}-1}
$$

where $\operatorname{Tr}$ is the matrix trace operator. The MUT complex permittivity can be evaluated iteratively by solving (7) and (5) using any two-dimensional numerical method [15].

\section{B. Sample-shifted method}

The sample-shifted method is also based on uncalibrated S-parameters, two-steps measurement setup and iterative calculation procedure for complex permittivity estimation. Firstly, the sample is positioned in the leftmost measurement cell and S-parameters are extracted for step (a). After, the sample is shifted to the rightmost measurement cell and S-parameters extraction repeated for step (b). In Fig. 3 is shown the sample-shifted setup with measurement steps highlighted. The MUT installed 
inside measurement cell must fulfill waveguide transversal section to avoid air gap regions between them, resulting in permittivity determination uncertainties [6].

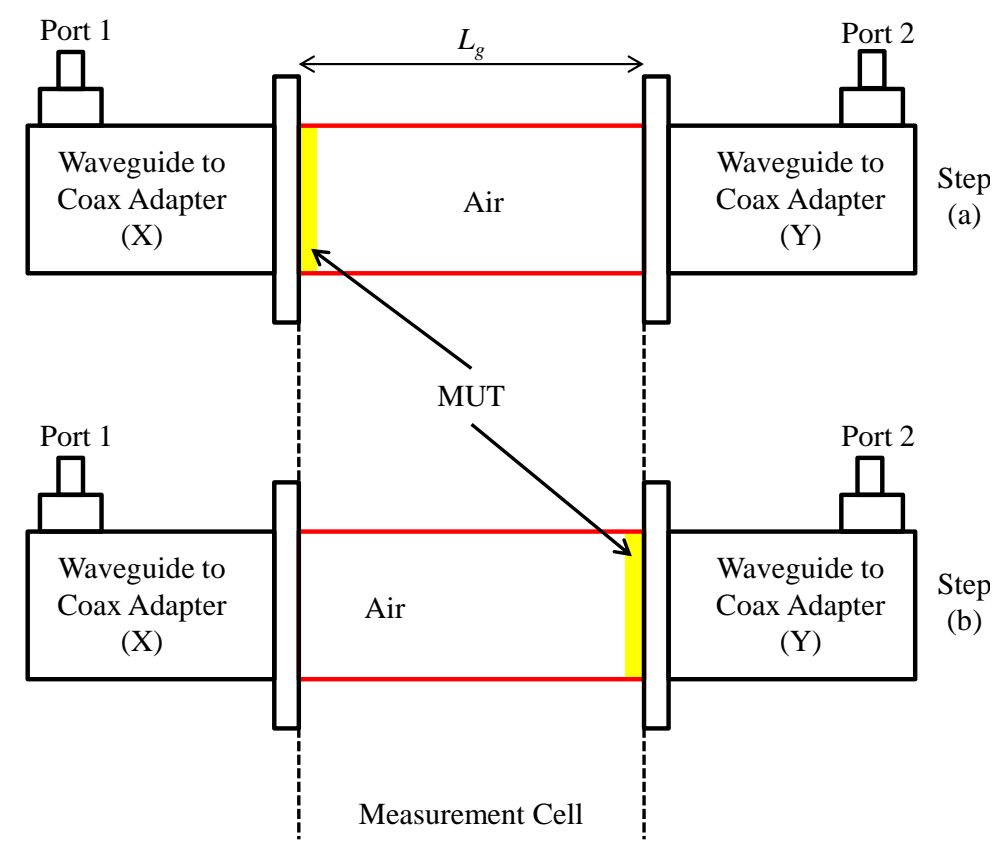

Fig. 3. The sample-shifted method setup.

One more time, it is assumed that only $\mathrm{TE}_{10}$ mode is guided through the measurement cell. The WCM matrices in Fig. 3 are described as:

$$
M_{a}=T_{X} T_{L} T_{G} T_{Y}, M_{b}=T_{X} T_{G} T_{L} T_{Y}
$$

where $T_{X}$ and $T_{Y}$ represent waveguide to coax transition, $T_{L}$ the sample-filled waveguide section and $T_{G}$ the air-region section (empty waveguide). The relation between the WCM matrices and measured raw S-parameters are presented in (2). For a measurement cell with isotropic and nonreflecting proprieties, the WCM matrix can be written as:

$$
T_{G}=\left[\begin{array}{cc}
\alpha & 0 \\
0 & 1 / \alpha
\end{array}\right], \alpha=\mathrm{e}^{-\gamma_{0}\left(L_{g}-L\right)}
$$

where $\gamma_{0}$ is the air-filled waveguide propagation constant defined in (3). The sample-filled waveguide section theoretical matrix $T_{L}$ is identical to the defined in air-region method (4) and first reflection and transmission coefficient equal to (5). The WCM matrices of waveguide to coax transitions $T_{X}$ and $T_{Y}$ will be eliminated during the calculation procedure.

Similar to air-region method, WCM matrices in (8) are combined in the following way:

$$
M_{b} M_{a}^{-1}=T_{X} T_{G} T_{L} T_{G}^{-1} T_{L}^{-1} T_{X}^{-1}
$$


where $T_{Y}$ is eliminated. According to (10), $M_{b} M_{a}^{-1}$ and $T_{G} T_{L} T_{G}{ }^{-1} T_{L}^{-1}$ are similar matrices and have the same trace. Combining (9), (4) and similar matrices trace in (10), theoretical and measured Sparameters can be related by:

$$
\left(\frac{\mathrm{T}-1 / \mathrm{T}}{\Gamma-1 / \Gamma}\right)^{2}=\frac{\operatorname{Tr}\left(M_{b} M_{a}^{-1}\right)-2}{\alpha^{2}+1 / \alpha^{2}-2}
$$

using (11) and (5), the MUT complex permittivity is determined by any two-dimensional numerical method.

\section{COMPOSITE MATERIAL CHARACTERIZATION}

The composite material electromagnetic characterization was performed for the L-band (0.96 to $1.45 \mathrm{GHz}$ ) and $\mathrm{C}$-band (3.3 to $4.9 \mathrm{GHz}$ ). Two waveguide to coax transitions and five sample holders are conceived for each frequency band; using the standard rectangular waveguides WR-770 and WR229 for the L-band and C-band, respectively. The sample holders are responsible for accommodate the MUT and form the measurement cell and extra cells for each characterization method. For L-band, sample holder is $12 \mathrm{~mm}$ thick and $8 \mathrm{~mm}$ for C-band. The manufactured prototypes for material characterization are shown in Fig. 4 for both frequency bands. The WR-770 waveguide to coax transitions are presented connected to five sample holders and WR-229 waveguide to coax transition connected to three sample holders.

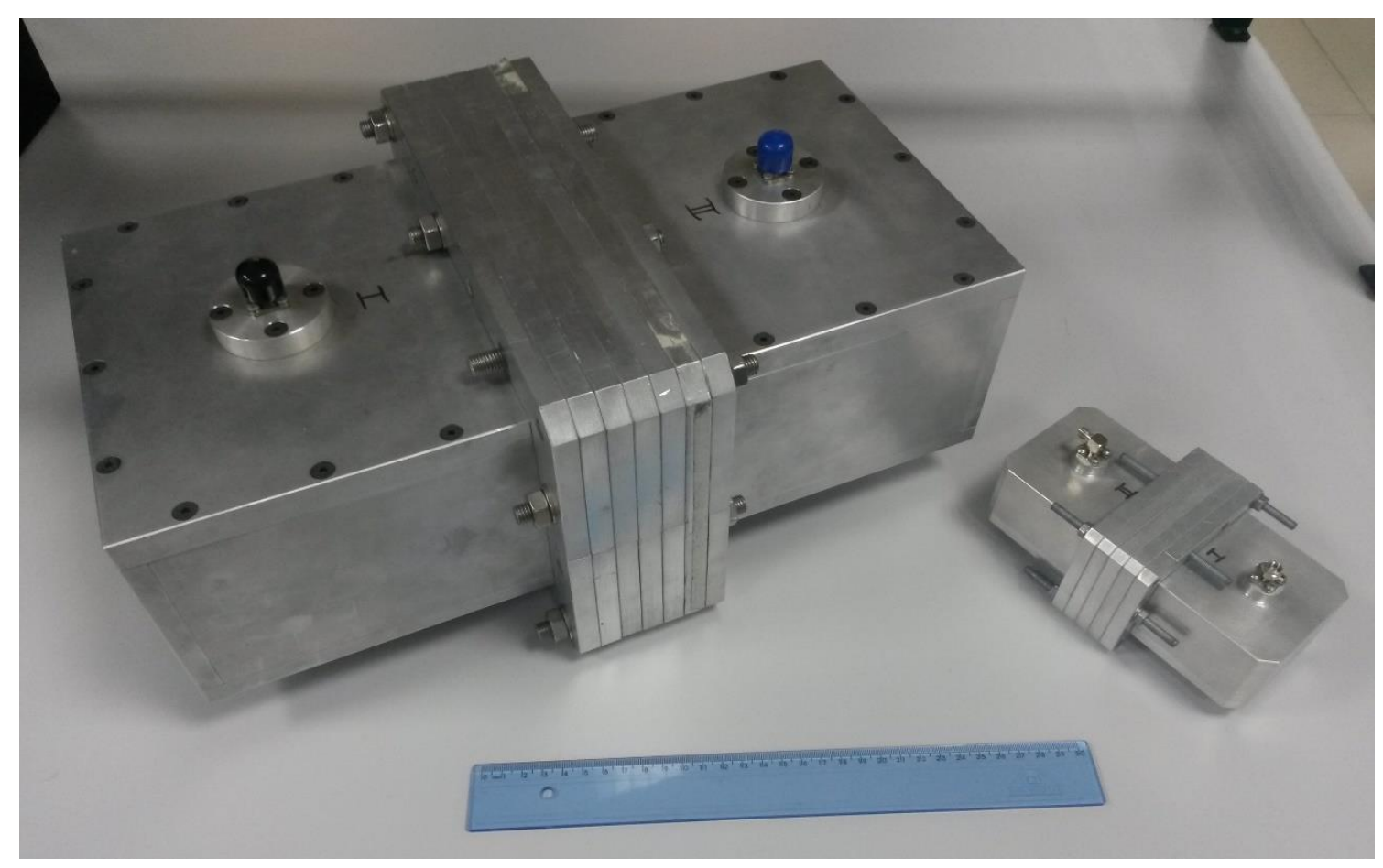

Fig. 4. Measurement setup for the composite material characterization. 
The two presented methods were implemented in the C-band material characterization for comparison purpose. However, in the L-band characterization only the sample-shifted method was applied, because of the sample holders' length that was not long enough to perform extra cells implementation for the air-region method. In material characterization measurements only a coaxial calibration type (short-open-load-thru) is performed, since sample-shifted and air-region methods are calibration independent solutions.

\section{A. Fiberglass composite}

Fiberglass composite (FGC) material is a dielectric material commonly used in aircraft structures and its dielectric constant and loss tangent knowledge is important and recommended for antenna integration on aircraft. In Fig.5 are presented the prepared FGC samples for characterization in L- and C-band. Additionally, one C-band sample holder is presented separately. The FGC samples are 2mm thick with transversal dimensions identical to correspondent rectangular waveguide setup (WR-229 and WR-770). To procedure the material characterization measurements, the samples are installed in one sample holder and the reaming ones used to create the measurement cells and extra cells, depending on implemented characterization method.

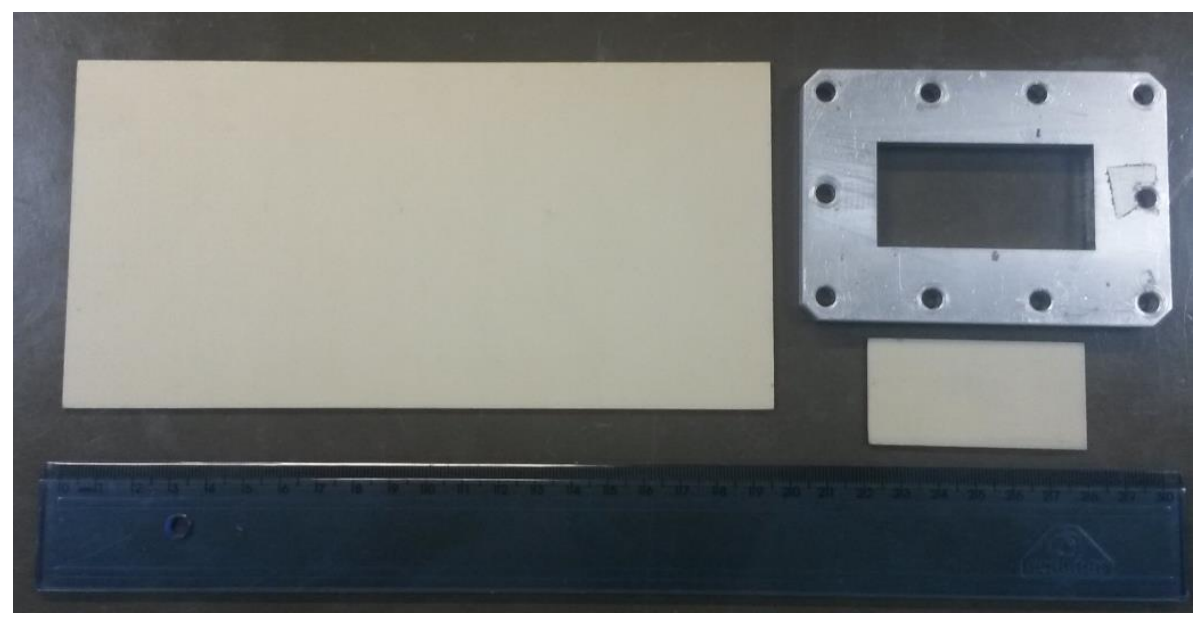

Fig. 5. FGC samples for L- and C-band characterization.

The FGC characterization in the C-band is performed for both presented methods. In air-region method, five sample holders are used, one for MUT installation, two for $L_{03}$ extra cell and other two for $L_{04}$ extra cell. Thus, air-region measurement cell is $8 \mathrm{~mm}$ and extra cells $L_{03}$ and $L_{04}$ are $16 \mathrm{~mm}$ thick. In sample-shifted method, only three sample holders are used, resulting in a measurement cell $L_{g}$ equal to $24 \mathrm{~mm}$. In the L-band FGC characterization, only sample shifted method is used. In this case, five sample holders are employed, resulting in a $60 \mathrm{~mm}$ longer measurement cell. Calibration 
independent methods determine MUT complex permittivity solving iteratively their objective functions. A Nelder-Mead simplex algorithm (as implemented in the Matlab routine fminsearch) is used to solve (7) for air-region method and (11) for sample-shifted method, with the purpose of obtaining the MUT complex permittivity.

Fig. 6 reports the FGC dielectric constant and loss tangent for all characterization methods in the Cband. The dielectric constant results are in good agreement for both methods, with an average value of 4.6. In the air-region method, the discrepancy for low frequencies results can be attributed to the reduced extra cells length. On the other hand, the loss tangent results obtained by the two methods are nearly constant and close to each other, with an average value of 0.02 . The L-band FGC characterization results are presented in Fig 7. Obtained dielectric constant and loss tangent values are in good agreement with the C-band ones, average values of 4.6 and 0.019 , respectively. Moreover, they are very close to the well-known microwave fiberglass dielectric materials, such as FR4.

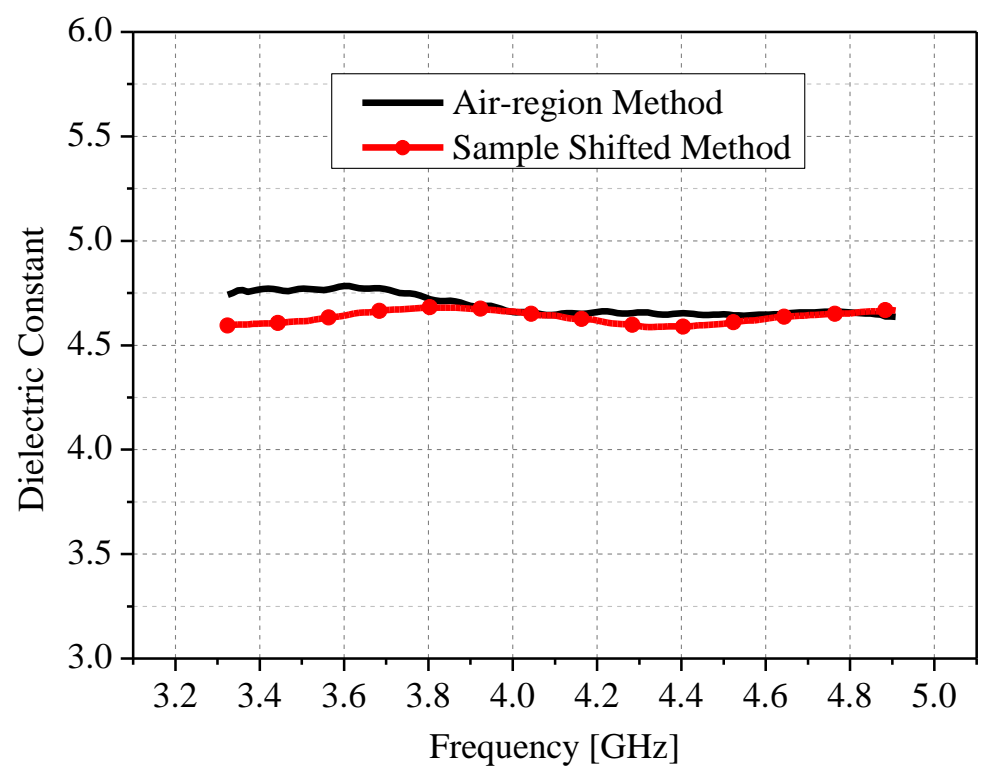

(a) Dielectric constant. 


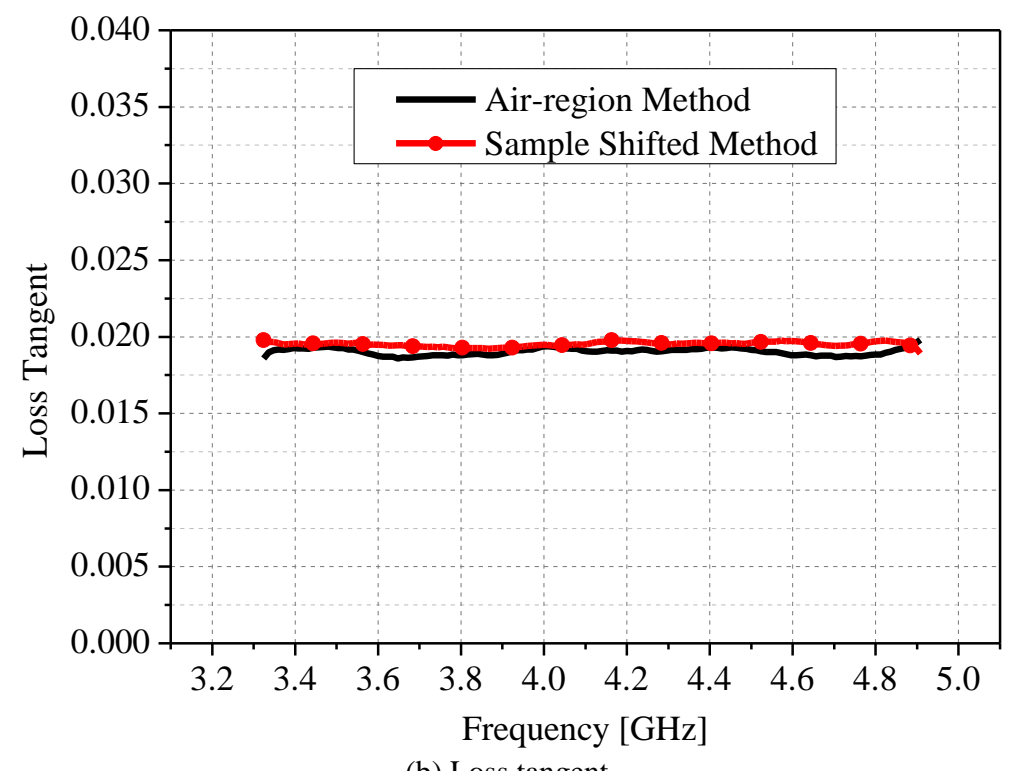

(b) Loss tangent.

Fig. 6. FGC complex permittivity in the C-band.

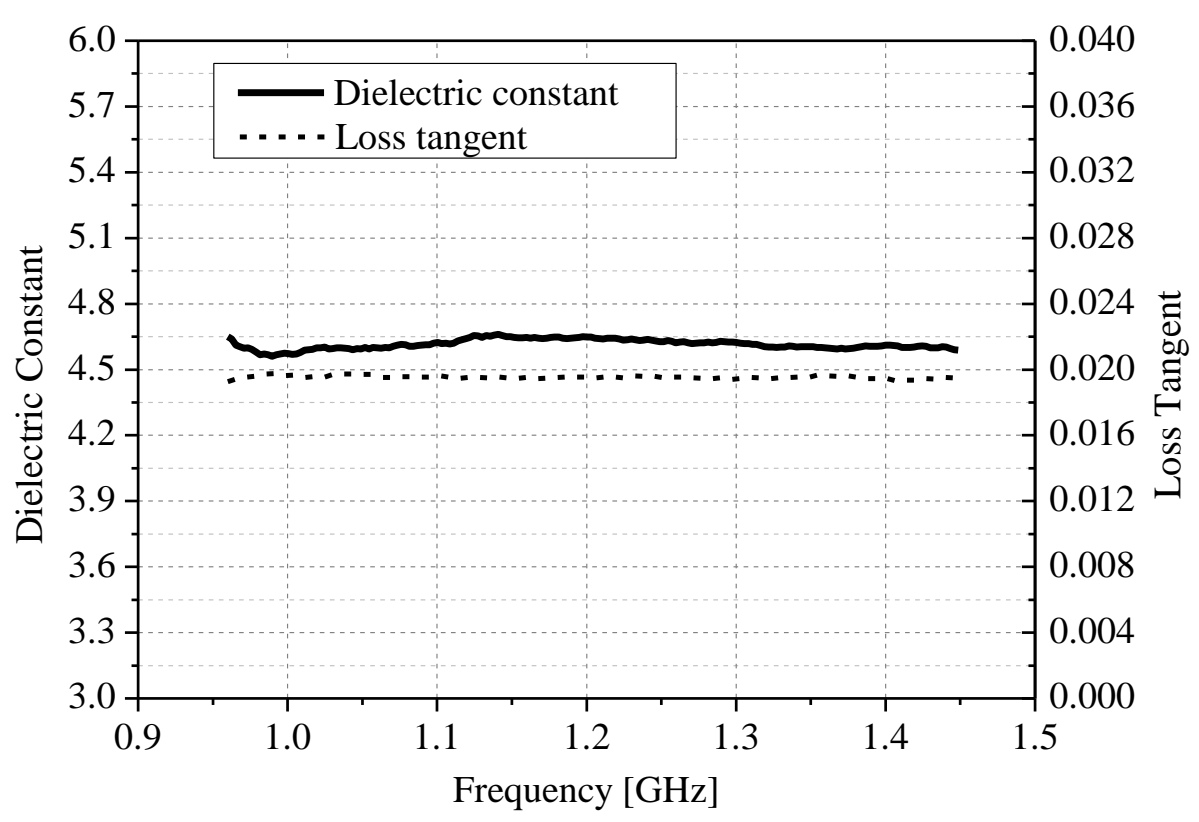

Fig. 7. FGC complex permittivity in the L-band.

In aircraft structures, the pure FGC material as presented in Fig. 5 is not generally used. In fact, the composite material undergoes to an adequacy process for aircraft operation requirements. Thus, the FGC is painted with a compound made of ink and substances as mica, varnish and others. For comparison purposes, pure FGC samples are painted with a composite ink as shown in Fig. 8 and characterized using sample shifted method in the L- and C-band. Again, sample shifted method is implemented with three sample holders for C-band and five sample holders for L-band, resulting in a $24 \mathrm{~mm}$ and $60 \mathrm{~mm}$ measurement cell, respectively. The painted FGC samples presented in Fig. 8 suffer from dimensions' imperfections and do not accommodated perfectly to the sample holder, which can result in complex permittivity determination uncertainties [6]. 


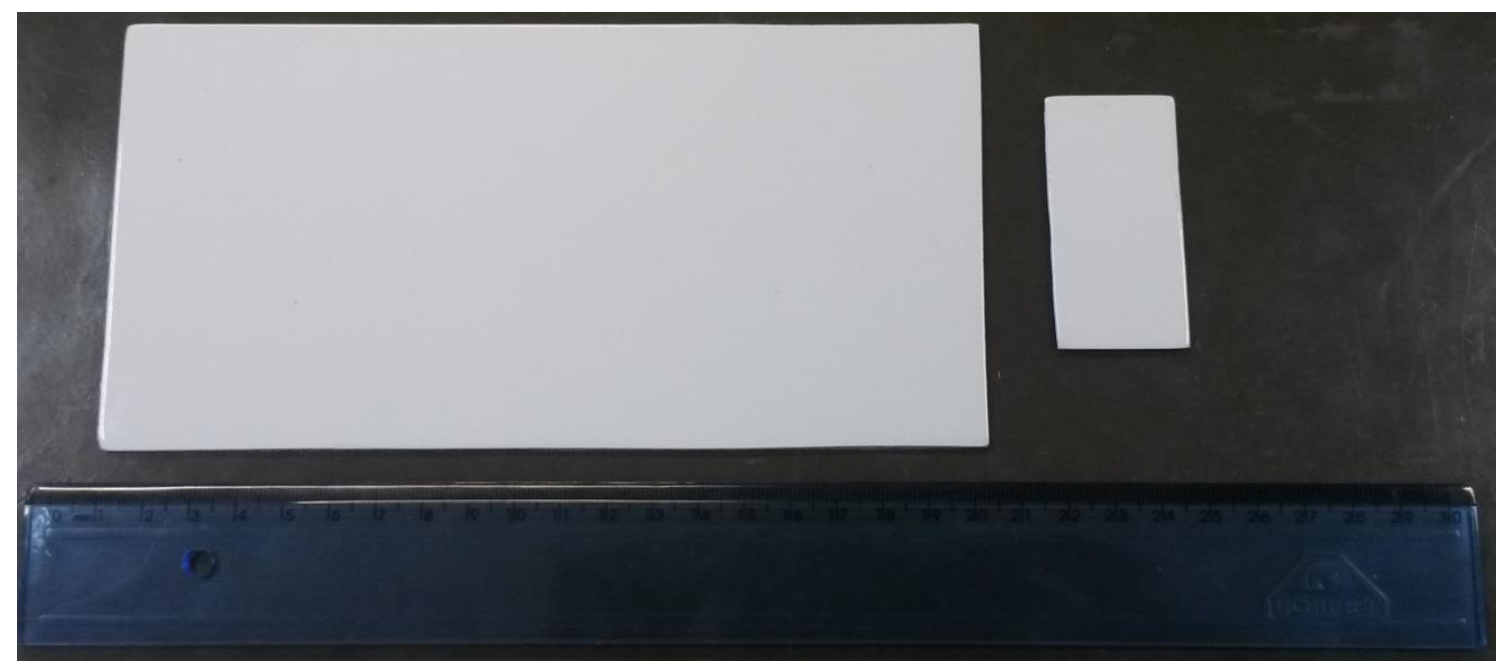

Fig. 8. Painted FGC samples for L- and C-band characterization.

The painted FGC characterization results for the C-band are reported in Fig. 9. According to Fig. 9, the painted FGC has a higher dielectric constant compared to pure FGC, but its obtained loss tangent is reduced due to MUT dimensions imperfection. The achieved dielectric constant and loss tangent mean values are 4.73 and 0.017 for painted FGC, respectively. Fig. 10 reports painted FGC characterization results for L-band. As presented for C-band, dielectric constant is increased and loss tangent reduced, compared to pure FGC results. For L-band, dielectric constant mean value is 4.85 and loss tangent 0.016 . Therefore, treated FGC for aircraft applications can have a modified complex permittivity compared to pure FGC.

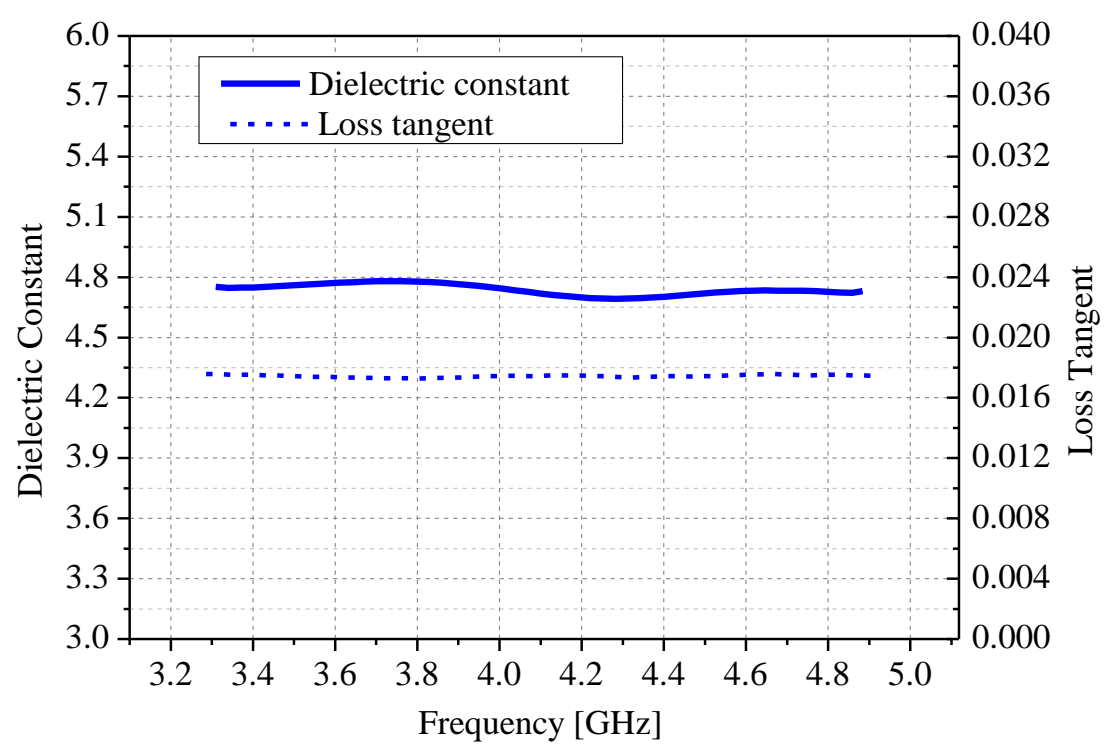

Fig. 9. Painted FGC complex permittivity in the C-band. 


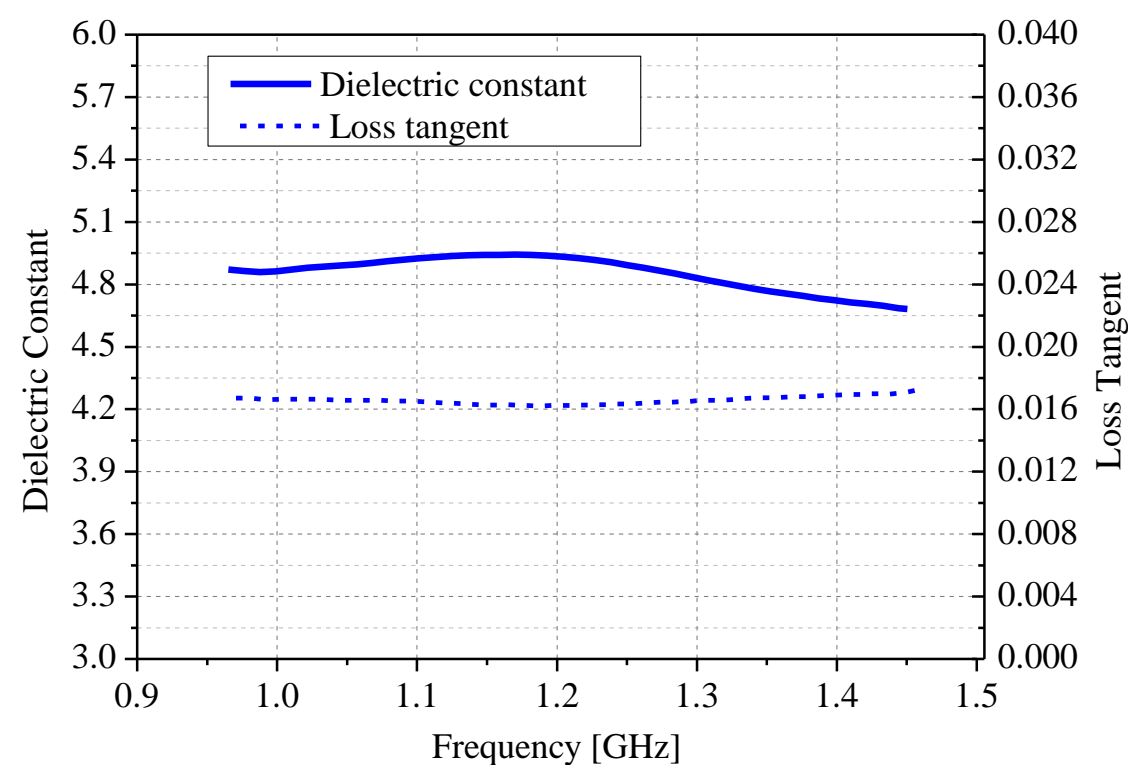

Fig. 10. Painted FGC complex permittivity in the L-band.

\section{B. Honeycomb}

Honeycomb is another dielectric material used in aircraft structures. As illustrated in Fig. 11, honeycomb is composed by some layers of fiberglass and air gaps. These samples are $7.65 \mathrm{~mm}$ thick with transversal dimensions equal to WR-770 and WR-229 sample holders.

The C-band characterization is conducted for sample-shifted and air-region methods. For the first, three sample holders are employed for measurement cell implementation and five sample holders for air-region method, as applied for FGC characterization. The honeycomb C-band results are presented in Fig. 12. The dielectric constant varies from 1.7 to 2.0 with an average value of 1.9 for air-region method, whereas sample-shifted result is 1.7 . For loss tangent, the obtained average values for airregion and sample-shifted method are 0.059 and 0.065 , respectively.

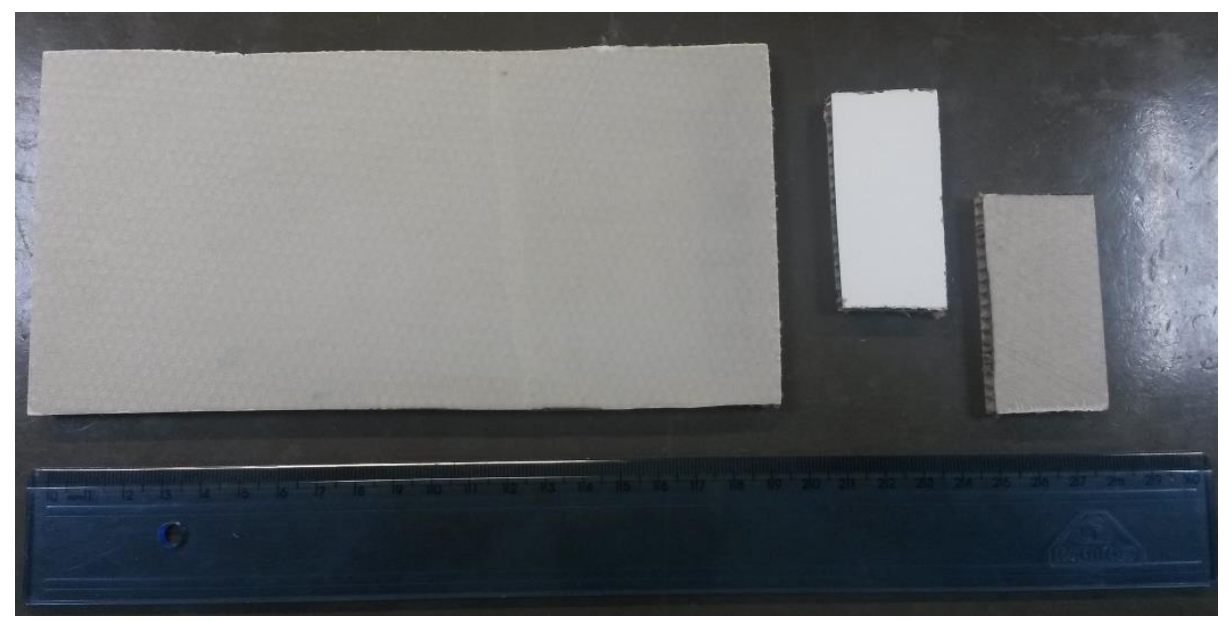

Fig. 11. Honeycomb samples for L- and C-band characterization. 


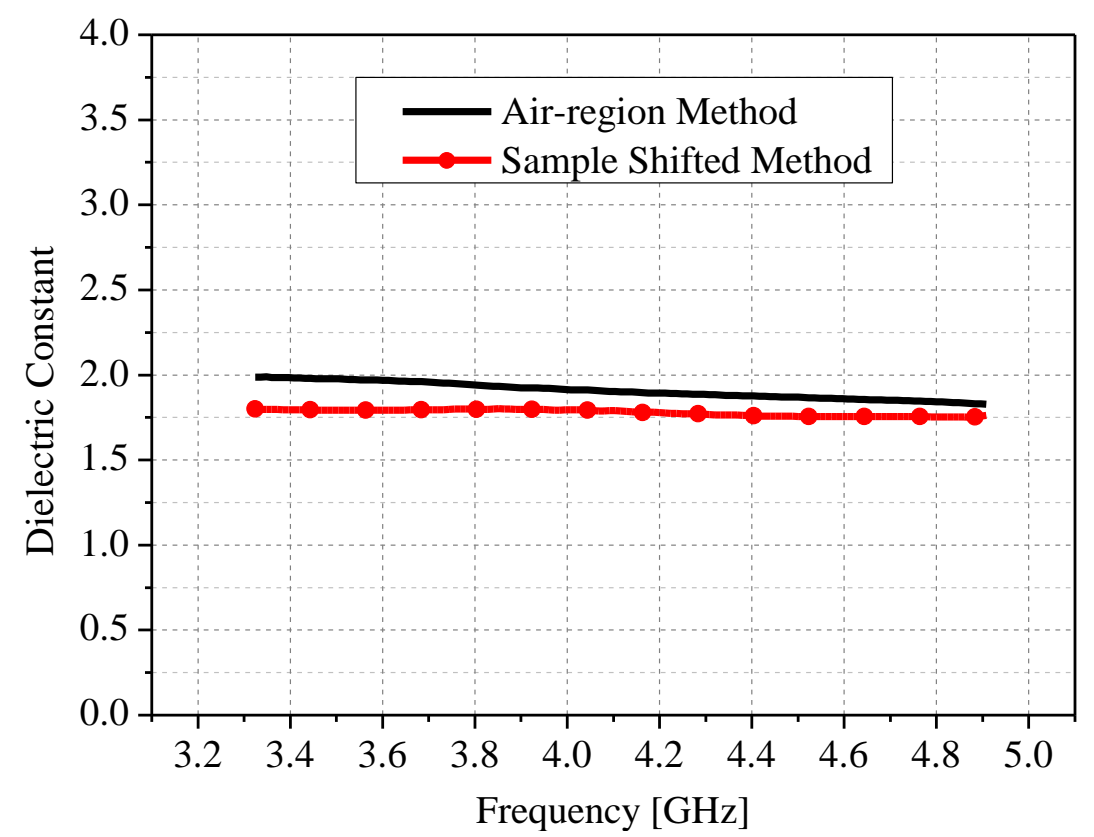

(a) Dielectric constant.

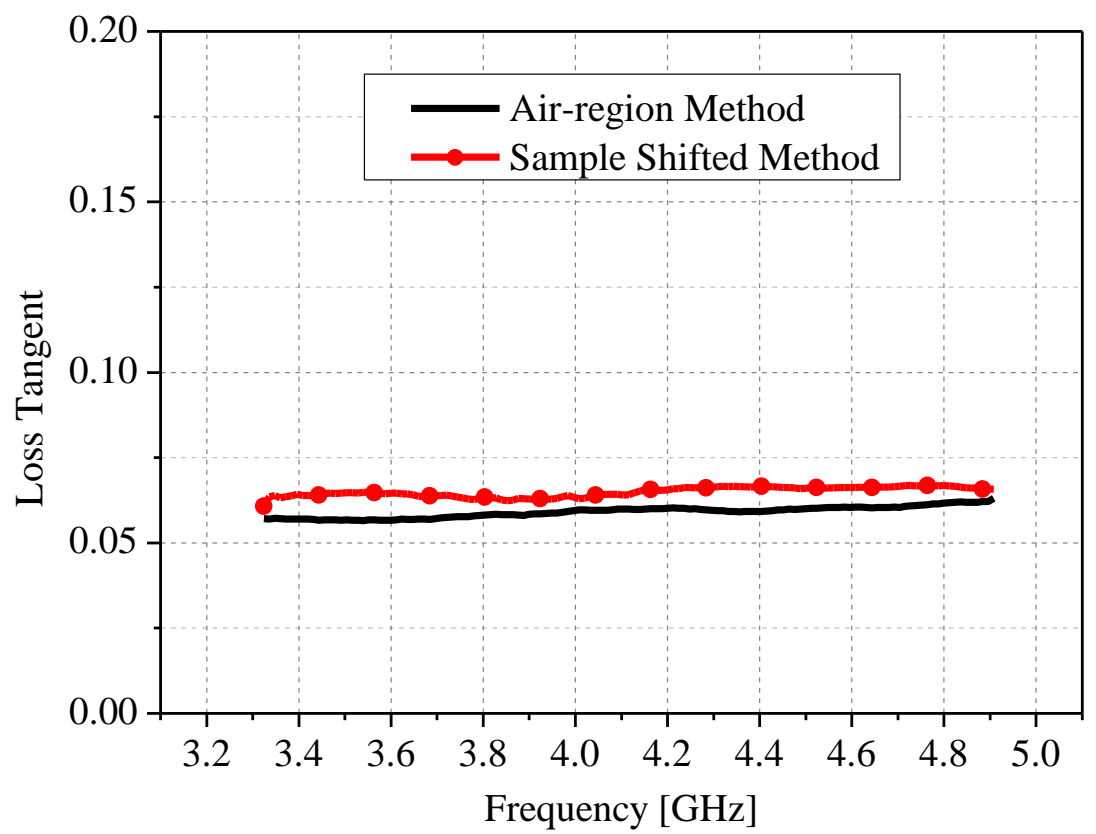

(b) Loss tangent.

Fig. 12. Honeycomb complex permittivity in the C-band.

The honeycomb characterization in L-band is performed with sample-shifted method implemented with five sample holders. In Fig. 13 are summarized the obtained results of the dielectric constant and loss tangent. The dielectric constant average value is approximately 1.9, which is in good agreement with the C-band results, whereas the average value of the loss tangent is 0.054 , which is lower than the C-band results. Nevertheless, L- and C-band results for honeycomb complex permittivity are considered consistent with those reported for similar materials [16] [17]. 


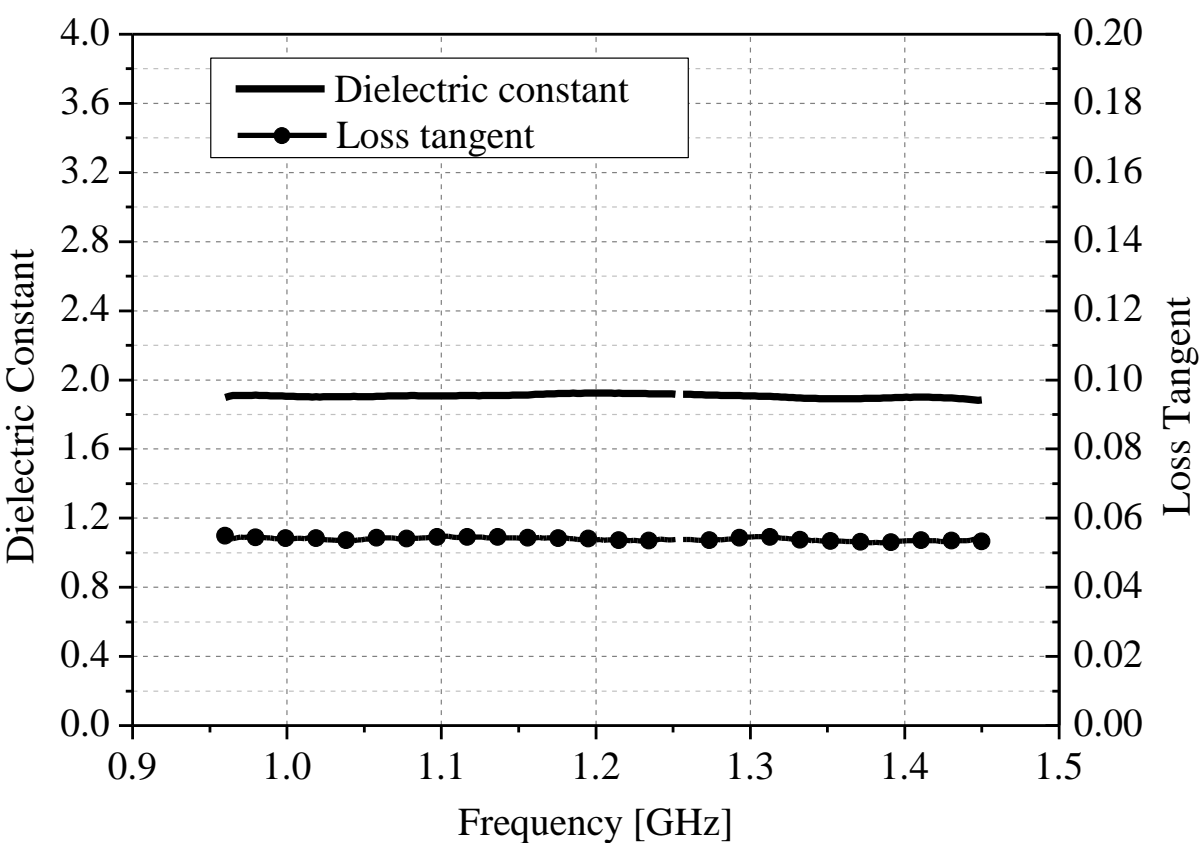

Fig. 13. Honeycomb complex permittivity in the L-band.

\section{NUMERICAL SIMULATIONS AND EXPERIMENTAL RESULTS}

Numerical simulations based on finite element method (FEM) and method of moments (MoM), as well as experiments in a semi-anechoic chamber, have been carried out in order to validate the material characterization presented in the previous section. The 3.0-meters full-scale prototype of an Embraer light jet aircraft is shown in Fig. 14. The dorsal fin model is composed by two dielectric materials, named FGC and honeycomb, supported by a section of the fuselage in aluminum. Fig. 14 (b) displays a photograph of the commercial aeronautical L-band antenna fixed to the aircraft dorsal fin. The dielectric constants and loss tangents of the fiberglass and honeycomb, obtained in the previous section, were used in the dorsal fin numerical model.

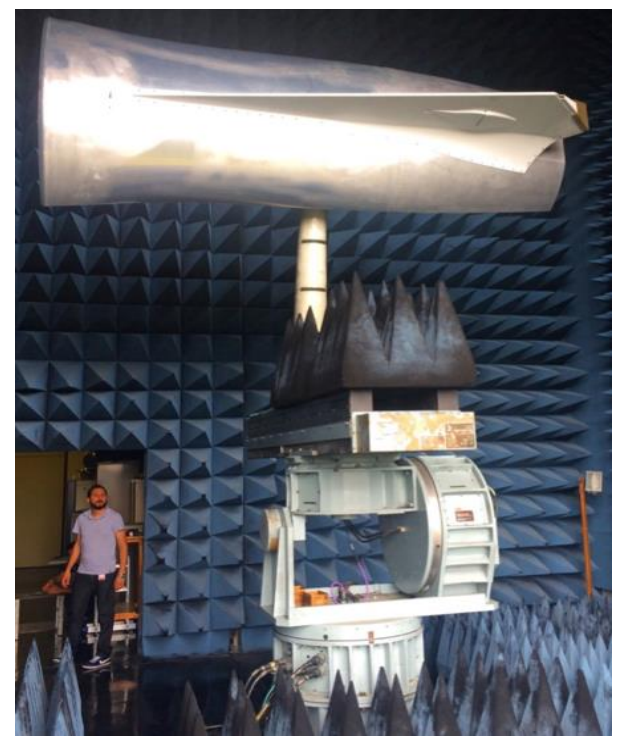

(a) Prototype in the semi-anechoic chamber.

Brazilian Microwave and Optoelectronics Society-SBMO Brazilian Society of Electromagnetism-SBMag

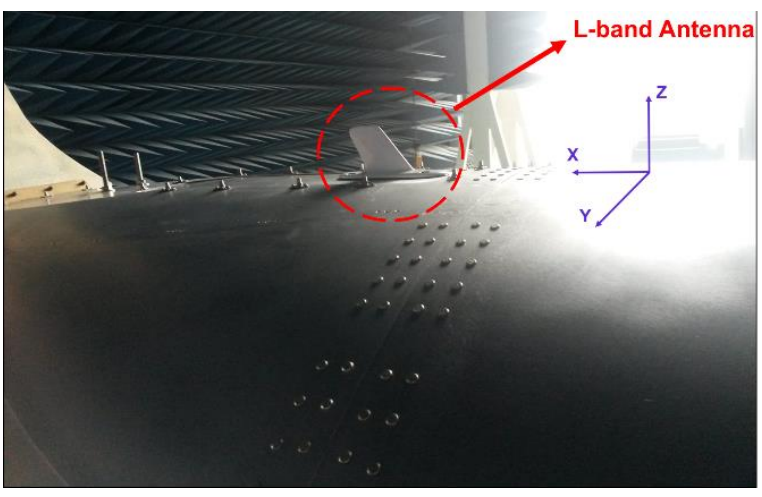

(b) The L-band antenna.

Received 30 Oct 2016; for review 03 Nov 2016; accepted 28 Dec 2016 (C) 2017 SBMO/SBMag 
Fig. 14. The 3.0-meters full-scale prototype of an Embraer light jet aircraft dorsal fin.

Two blade antennas, for VHF and L-band, have been evaluated. Since, there is little or no information about the antenna geometry, two equivalent models of the antenna under test (AUT) had to be devised for carrying out the numerical analysis. The equivalent antenna models consist of a trapezoidal metallic sheets with some slots to increase their bandwidth. The AUT is positioned on the section of the fuselage, so that the composite material is able to fully cover it. Fig. 15 reports a comparison between the numerical simulations and experimental results of the L-band antenna radiation pattern at $1.1 \mathrm{GHz}$. It is observed an excellent agreement, demonstrating the material characterization provided an accurate prediction of the composite material properties. The measurements presented in Fig. 16 demonstrates the cover made by FGC or honeycomb materials have insignificant impact in the L-band antenna performance, as predicted in the material characterization. The radiation pattern kept its omnidirectional feature, as well as its overall shape (Fig. 16 (a)). Complimentary, the VSWR from 0.95 to $1.2 \mathrm{GHz}$ is approximately the same with and without fairing, as illustrated in Fig. 16(b).

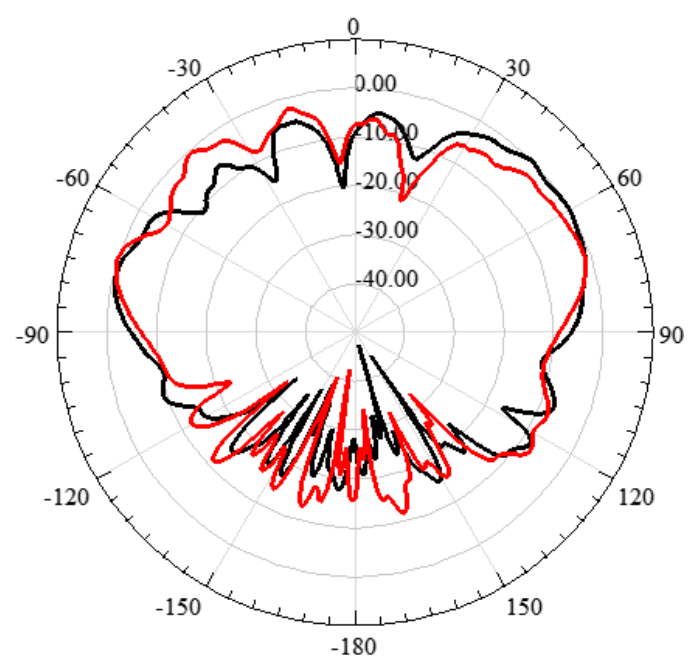

(a) $\phi=0^{\circ}(\mathrm{XZ}$ plane $)$ 


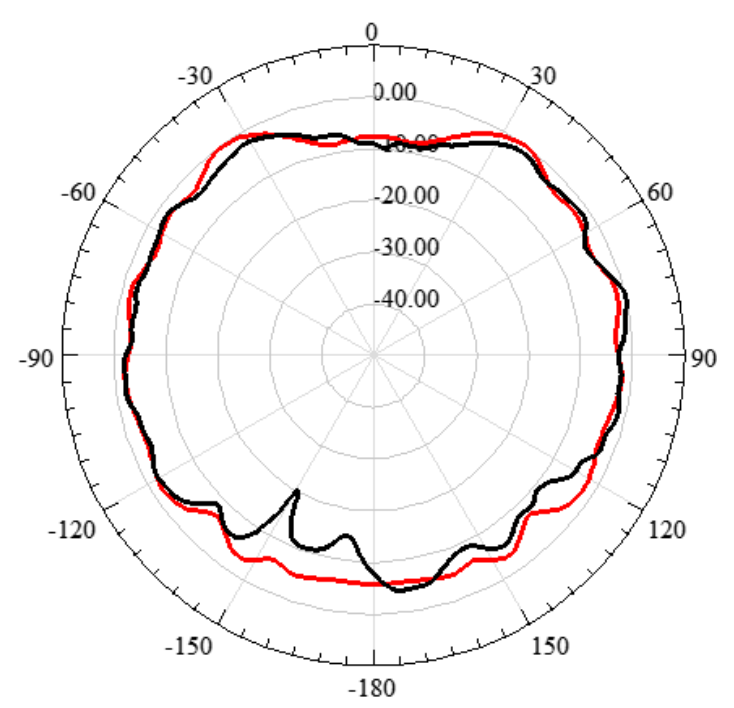

(b) $\theta=90^{\circ}(\mathrm{XY}$ plane $)$

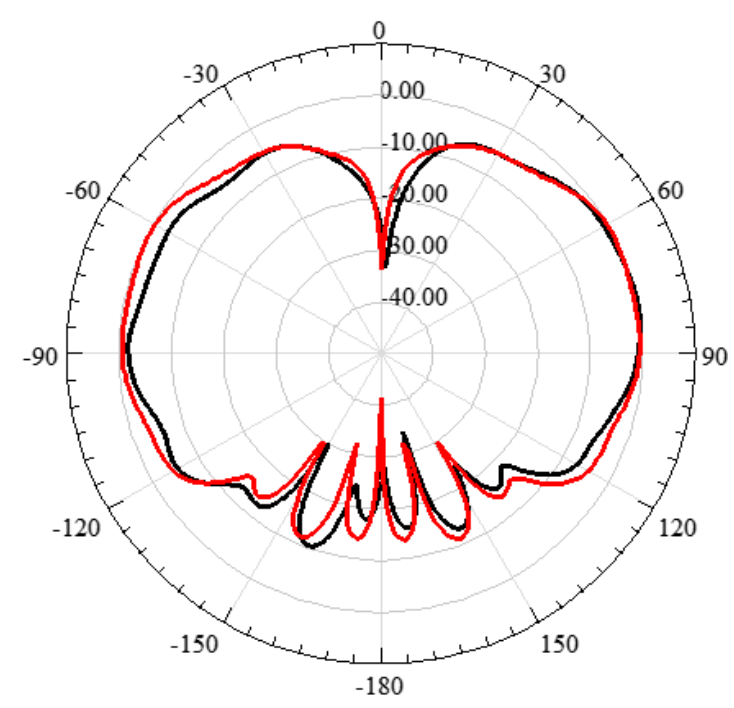

(c) $\phi=90^{\circ}$ (YZ plane)

Fig. 15. L-band antenna radiation pattern at 1.1GHz: simulations (red curves); measurements (black curves).

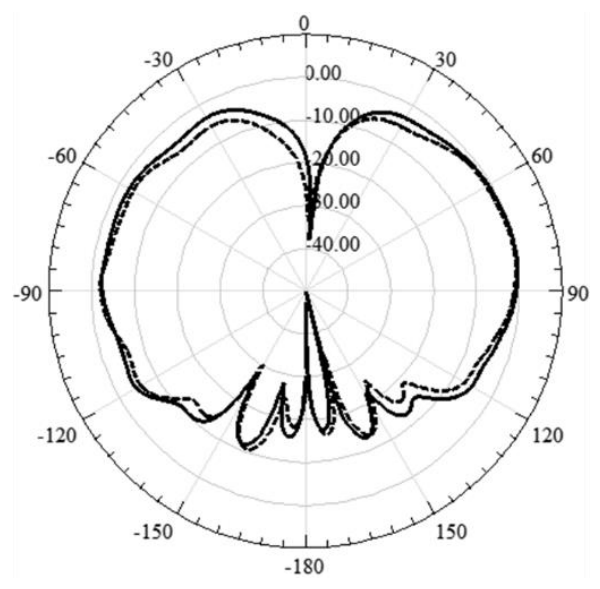

(a) $\phi=90^{\circ}$ (YZ plane): with fairing (continuous curve) and without fairing (dashed curve).

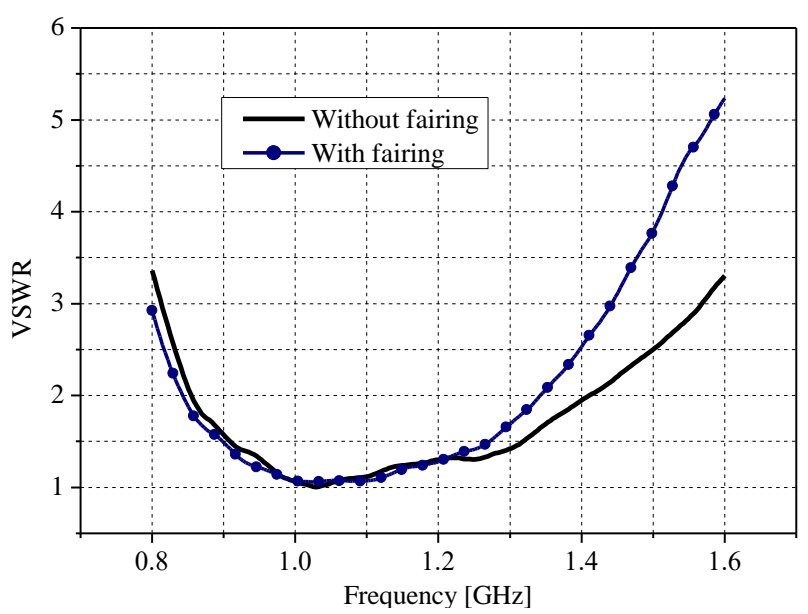

(b) VSWR

Fig. 16. L-band antenna experimental performance analyses at $1.1 \mathrm{GHz}$.

We have carried out the same performance analyses for a commercial VHF blade antenna, operating at $250 \mathrm{MHz}$. Fig. 17 presents comparison between simulations and experiments of the antenna radiation pattern in the three main planes, whereas Fig. 18 reports the obtained results of the radiation pattern in the YZ plane and VSWR with and without the fairing. Once again, an excellent agreement is observed in all cases, confirming the success of the electromagnetic characterization not only in L-band, but also in VHF. 


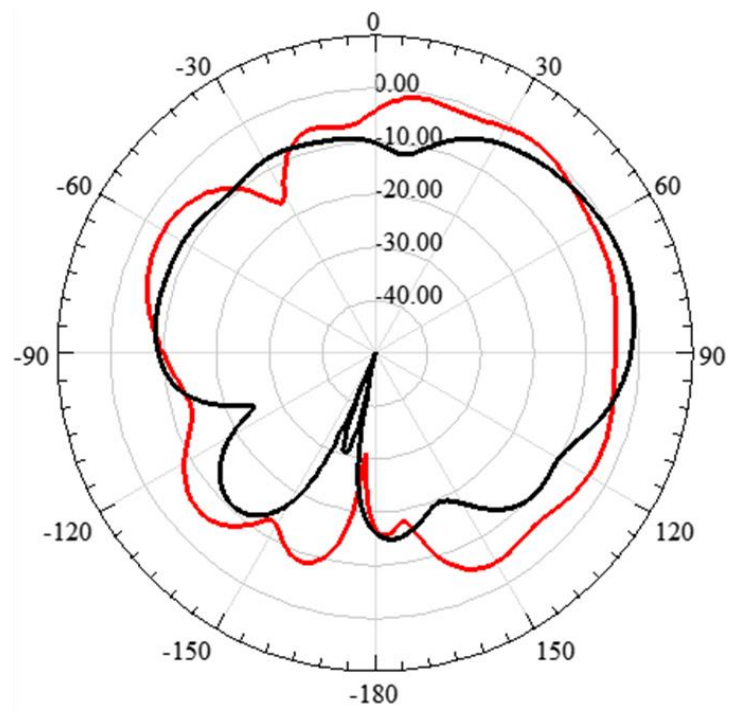

(a) $\phi=0^{\circ}(\mathrm{XZ}$ plane $)$

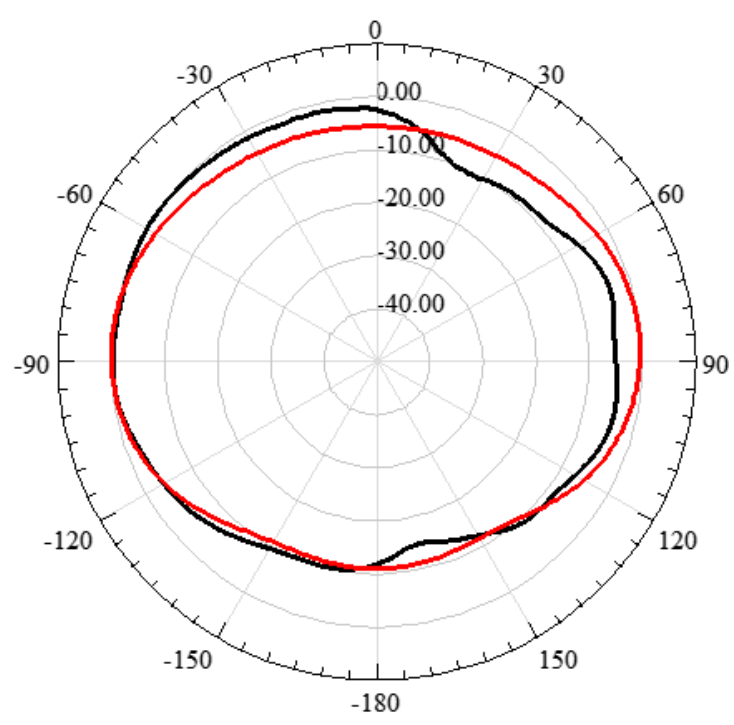

(b) $\theta=90^{\circ}(\mathrm{XY}$ plane $)$

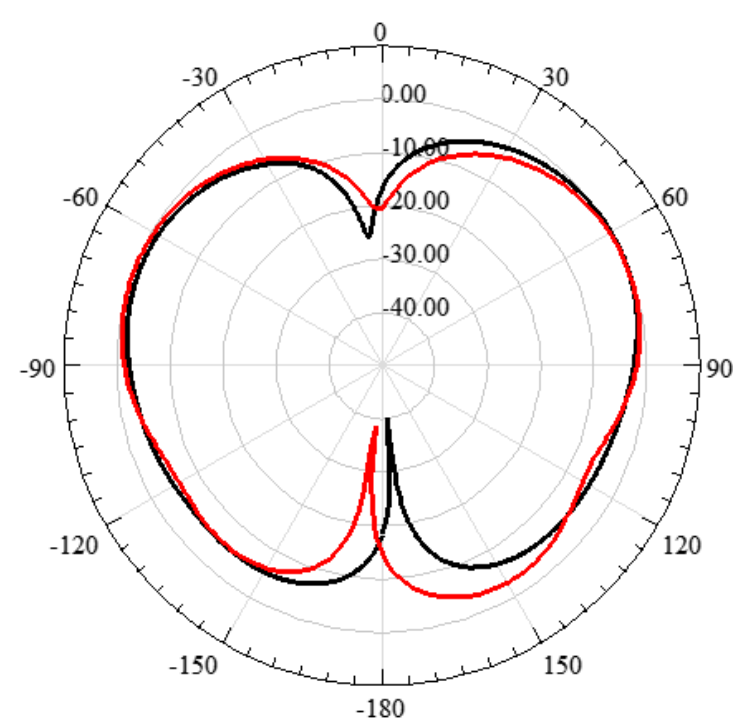

(c) $\phi=90^{\circ}$ (YZ plane)

Fig. 17. VHF antenna radiation pattern at $250 \mathrm{MHz}$ : simulations (red curves); measurements (black curves). 


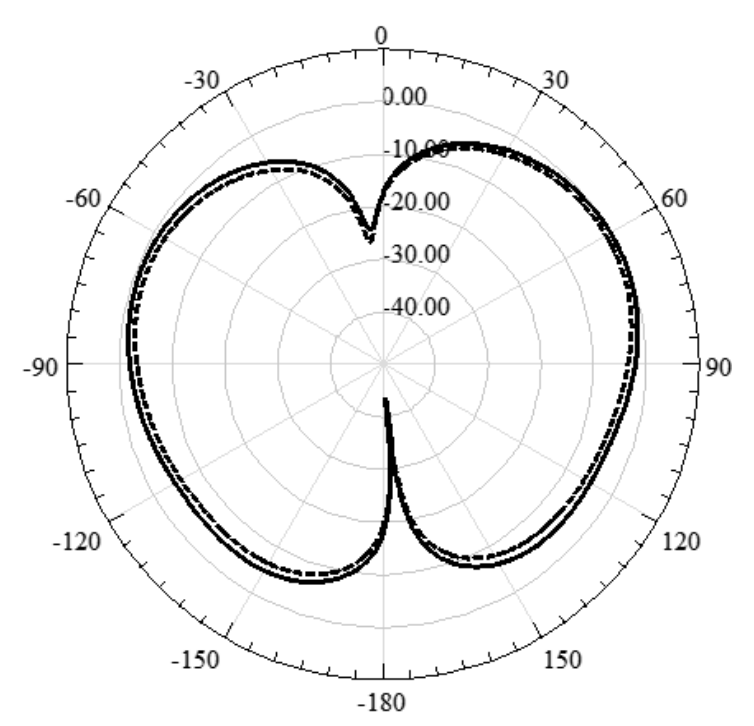

(a) $\phi=90^{\circ}$ (YZ plane): with fairing (continuous curve) and without fairing (dashed curve).

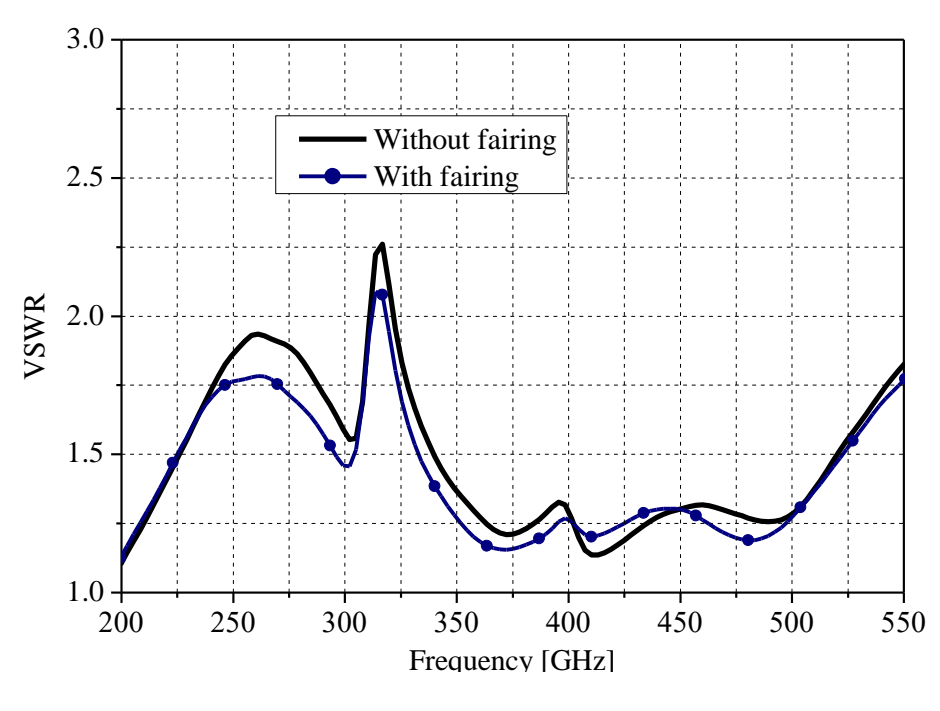

(b) VSWR

Fig. 18.VHF antenna experimental performance analyses at $250 \mathrm{MHz}$.

\section{CONCLUSIONS}

Two different uncalibrated S-parameters methods have been efficiently applied to predict the electromagnetic properties of composite materials, typically used in aircraft structures, namely fiberglass and honeycomb. Dielectric constants of 4.6 and 1.84 and loss tangent of 2.0x10-2 and 6.1 x10-2 have been obtained for fiberglass and honeycomb composite materials, respectively.

A $3.0 \mathrm{~m}$ prototype of an Embraer light jet aircraft dorsal fin have been fabricated and used in the experiments, with the purpose of investigating the impact of installing blade antenna on fuselage made of composite materials. The influence of the analyzed fiberglass-based composite materials on the embedded aeronautical antenna figures of merit has been considered extremely small, since its performance showed no significant changes. Furthermore, the simulation results agreed with the experimental ones with excellent accuracy, validating the proposed material characterization. Future works regards the material characterization in the X-band.

\section{ACKNOWLEDGMENTS}

This work was partially supported by Finep/Funttel Grant No. 01.14.0231.00, under the Radio Communications Reference Center (CRR) project of the National Institute of Telecommunications (Inatel), Brazil. Authors also thank the financial support from CNPq, CAPES, MCTI and FAPEMIG and technical support from Anritsu, Keysight, and ESSS-ANSYS.

\section{REFERENCES}

[1] L. F. Chen, C. K. Ong, C. P. Neo, V. V. Varadan, and V. K. Varadan, Microwave Electronics: Measurement and Materials Characterization, Hoboken, NJ: Wiley, 2004.

[2] U. C. Hasar, "A new calibration-independent method for complex permittivity extraction of solid dielectric materials," IEEE Microw. Wireless Compon. Lett., vol. 18, no. 12, pp. 788-790, Dec. 2008. 
[3] U. C. Hasar and O. Simsek, "A calibration-independent microwave method for position-insensitive and nonsingular dielectric measurements of solid materials," J. Phys. D: Appl. Phys., vol. 42, no. 7, Mar. 2009.

[4] A. M. Nicolson and G. F. Ross, "Measurement of the intrinsic properties of materials by time-domain techniques," IEEE Trans. Instrum. Meas., vol. 19, no. 4, pp. 377-382, Nov. 1970.

[5] W. B. Weir, "Automatic measurement of complex dielectric constant and permeability at microwave frequencies," Proc. IEEE, vol. 62, no. 1, pp. 33-36, Jan. 1974.

[6] J. Baker-Jarvis, "Transmission/reflection and short-circuit line permittivity measurements," NIST, Boulder, CO, Tech. Note 1341, Jul. 1990.

[7] A. H. Boughriet, C. Legrand, and A. Chapoton, "A noniterative stable transmission/reflection method for low-loss material complex permittivity determination,” IEEE Trans. Microw. Theory Tech., vol. 45, no. 1, pp. 52-57, Jan. 1997.

[8] J. Baker-Jarvis, E. J. Vanzura, and W. A. Kissick, "Improved technique for determining complex permittivity with the transmission reflection method," IEEE Trans. Microw. Theory Techn., vol. 38, no. 8, pp. 1096-1103, Aug. 1990.

[9] K. Chalapat, K. Sarvala, J. Li, and G. S. Paraoanu, "Wideband reference plane invariant method for measuring electromagnetic parameters of materials," IEEE Trans. Microw. Theory Tech., vol. 57, no. 9, pp. 2257-2267, Aug. 2009.

[10] U. C. Hasar and O. E. Inan, "A position-invariant calibration-independent method for permittivity measurements," Microw. Opt. Technol. Lett., vol. 51, no. 6, pp. 1406-1408, Jun. 2009.

[11] C. Wan, B. Nauwelaers, W. De Raedt, and M. Van Rossum, "Two new measurement methods for explict determination of complex permittivity,” IEEE Trans. Microw. Theory Tech., vol. 46, no. 11, pp. 1614-1619, Nov. 1998.

[12] M. D. Janezic, "Complex permittivity determination from propagation constant measurements," IEEE Microw. Guided Wave Lett., vol. 9, no. 2, pp. 76-78, Feb. 1999.

[13] C. Wan , B. Nauwelaers, W. De Raedt and M. Van Rossum, "Complex permittivity measurement method based on asymmetry of reciprocal two-ports", Electron. Lett., vol. 32, no. 16, pp. 1497-1498, Aug. 1996.

[14] G. B. Arfken and H. J. Weber, Mathematical methods for physicists, Burlington, MA: Elsevier, 2005.

[15] W. H. Press, S. A. Teukolsky, W. T. Vetterling, and B. P. Flannery, Numerical recipes in C: The art of scientific computing, Cambridge: Cambridge university press, 1992.

[16] F. C. Smith, "Effective permittivity of dielectric honeycombs ", Proc. Inst. Elect. Eng., Microw., Antennas, Propagat., vol. 146 , no. 1 , pp. $55-59,1999$.

[17] C. You, M. M. Tentzeris, and W. Hwang, "Multilayer Effects on Microstrip Antennas for Their Integration With Mechanical Structures", IEEE Trans. Antennas Propag., vol. 55, no. 4, pp. 1051-1058, Apr. 2007.

[18] Sensor Systems Inc. S65-5366-L series, Available in: 〈http://www.sensorantennas.com/product/l-band-antenna-9/>. Access at: October 12, 2016. 